\title{
Evaluation of lung transplant perfusion using iodine maps from novel spectral detector computed tomography
}

\author{
Nils Große Hokamp1,2, Amit Gupta1,2 \\ 'Department of Radiology, University Hospitals Cleveland Medical Center, ${ }^{2}$ Department of Radiology, Case School of Medicine, \\ Case Western Reserve University, Cleveland, OH, USA \\ Correspondence: Dr. Nils Große Hokamp, Case Western Reserve University, Department of Radiology, 11000 Euclid Ave, Cleveland, OH, \\ 44106, USA. E-mail: nils.grosse-hokamp@uk-koeln.de
}

\section{Abstract}

We report the case of a 51-year-old patient who underwent bilateral lung transplantation and presented with an unstable condition and sepsis 6 days after transplantation. The performed contrast enhanced spectral detector computed tomography (CT) using a dual-layer detector showed absence of perfusion in the left lung on iodine maps, although branches of the pulmonary artery were patent. This prompted retrospective evaluation of CT images and total venous occlusion of the left pulmonary veins was found. Here, iodine maps helped in raising conspicuity of loss of lung perfusion.

Key words: Dual energy computed tomography; iodine-maps; lung transplantation; spectral detector computed tomography; quantitative imaging

\section{Introduction}

We demonstrate a case of a patient with pulmonary venous occlusion after lung transplantation. Venous occlusion is a well-known, however, infrequent complication after lung transplantation, which was found in this case with the help of image reconstructions from novel spectral detector computed tomography (SDCT). ${ }^{[1]}$ SDCT allows for reconstruction of iodine maps and Z-effective $\left(Z_{\text {eff }}\right)$ maps that depend on effective atomic number and are color-coded. ${ }^{[2,3]}$ While color-coding is considered helpful in visual discrimination of structures, iodine maps allow for visualization and quantification of contribution of iodinated contrast media; ${ }^{[4]}$ due to their quantitative characteristics they can serve as a measure for organ perfusion. ${ }^{[2,5]}$

\begin{tabular}{|l|l|}
\hline \multicolumn{2}{|c|}{ Access this article online } \\
\hline Quick Response Code: & \\
\cline { 1 - 2 } & Website: \\
\hline & www.ijri.org \\
& DOI: \\
&
\end{tabular}

\section{Case History}

A 51-year-old patient with idiopathic pulmonary fibrosis underwent bilateral lung transplantation. In the first day after surgery, there was an unusual high oxygen demand and signs of pulmonary edema on chest X-rays especially in the left lung attributed to a primary graft dysfunction (score of two). Follow-up computed tomography (CT) of the chest on day three demonstrated patchy airspace opacities within left lung and imaging differentials included infection and early graft rejection. Starting on day 4, leucocyte counts increased and aggressive antibiotics treatment was started. But, the patient continued to worsen with increasing oxygen demands which warranted a computed

This is an open access journal, and articles are distributed under the terms of the Creative Commons Attribution-NonCommercial-ShareAlike 4.0 License, which allows others to remix, tweak, and build upon the work non-commercially, as long as appropriate credit is given and the new creations are licensed under the identical terms.

For reprints contact: reprints@medknow.com

Cite this article as: Große Hokamp N, Gupta A. Evaluation of lung transplant perfusion using iodine maps from novel spectral detector computed tomography. Indian J Radiol Imaging 2018;28:436-8. 
tomography-angiography (CTA) of the chest on day 6 with suspicion for pulmonary thromboembolism.

CTA demonstrated significant interval worsening of left lung including extensive consolidative opacities with air bronchograms involving the entire left lung as well as small right pleural effusion [Figure 1]; however, the central pulmonary arteries were found patent (yellow arrow in Figure 1). Since this pulmonary angiographic examination was carried out using a SDCT scanner, iodine-maps were available, which clearly demonstrated absence of perfusion in the left-sided lung [Figure 2]. Besides clear visualization of the discrepancy in lung perfusion, region of interest-based measurements within the iodine maps allowed for a quantification of this mismatch $(0.2$ and $1.0 \mathrm{mg} / \mathrm{mL}$ in the left and right lung, respectively, Figure 2). The mismatch was visually even more impressive on $\mathrm{Z}_{\text {eff }}$ images [Figure 2]. In light of added information from novel maps from SDCT, repetitive analysis of $\mathrm{CT}$ images was performed, and venous occlusion of the left-sided pulmonary veins was found [Figure 3]. Patient underwent left pneumonectomy on postoperative day 9 . Intraoperatively, the left side bronchial

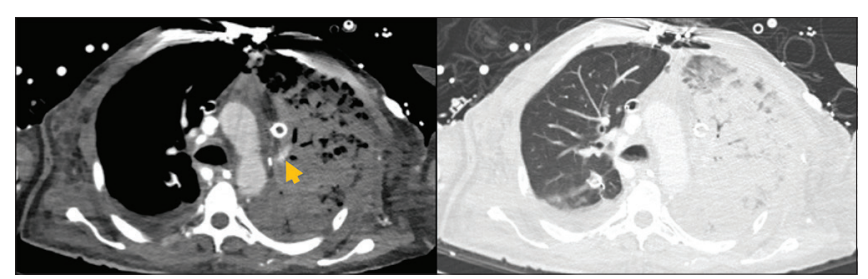

Figure 1: Conventional computed tomography (CT) image reconstructions of the imaging study on day six. Conventional, axial CT images in soft tissue (left) and lung window showing extensive consolidative opacification of the left lung. Yellow arrow indicates a patent central pulmonary arterial branch anastomosis looked intact with some granulation tissue, while there was significant fluid, pus, and debris on left upper lobe and left lower lobe orifice. The lung appeared consolidated not viable. Histologically, there were findings consistent with extensive hemorrhagic infarction of the left lung.

\section{Discussion}

While single-energy of conventional CT measures the global phenomenon of attenuation of any examined object, the attenuation properties of low- and high-energy photons are assessed separately in dual-energy CT. ${ }^{[3]}$ Since the attenuation coefficient of any given material is energy dependent, the acquired information allows for material separation, for example, for iodine resulting in iodine maps. These maps demonstrate quantitative characteristics and therefore can serve as measures of organ perfusion.

Most common vascular complications after transplantation include acute organ rejection, eventually resulting thrombosis of supplying arteries or draining veins. Rejection of the donor organ (graft) by the host's immune system is one of the most common risks and complication of any transplantation. ${ }^{[6]} \mathrm{Graft}$ rejection is categorized with respect to its timely occurrence into either hyper acute, acute, and chronic. ${ }^{[6]}$ Hyper acute organ rejection occurs immediately following transplantation and is caused by pre-existing antibodies again surface structures of the graft, for example, rhesus incompatibility. ${ }^{[6,7]}$ However, acute organ rejection is T-cell mediated and results in inflammation with leukocyte migration; this may result in cell swelling that impairs the graft's perfusion. ${ }^{[7,8]}$ Acute organ rejection usually occurs within weeks to months after transplantation. ${ }^{[6]}$ Chronic organ rejection is also T-cell mediated, however, due to different mechanisms, which
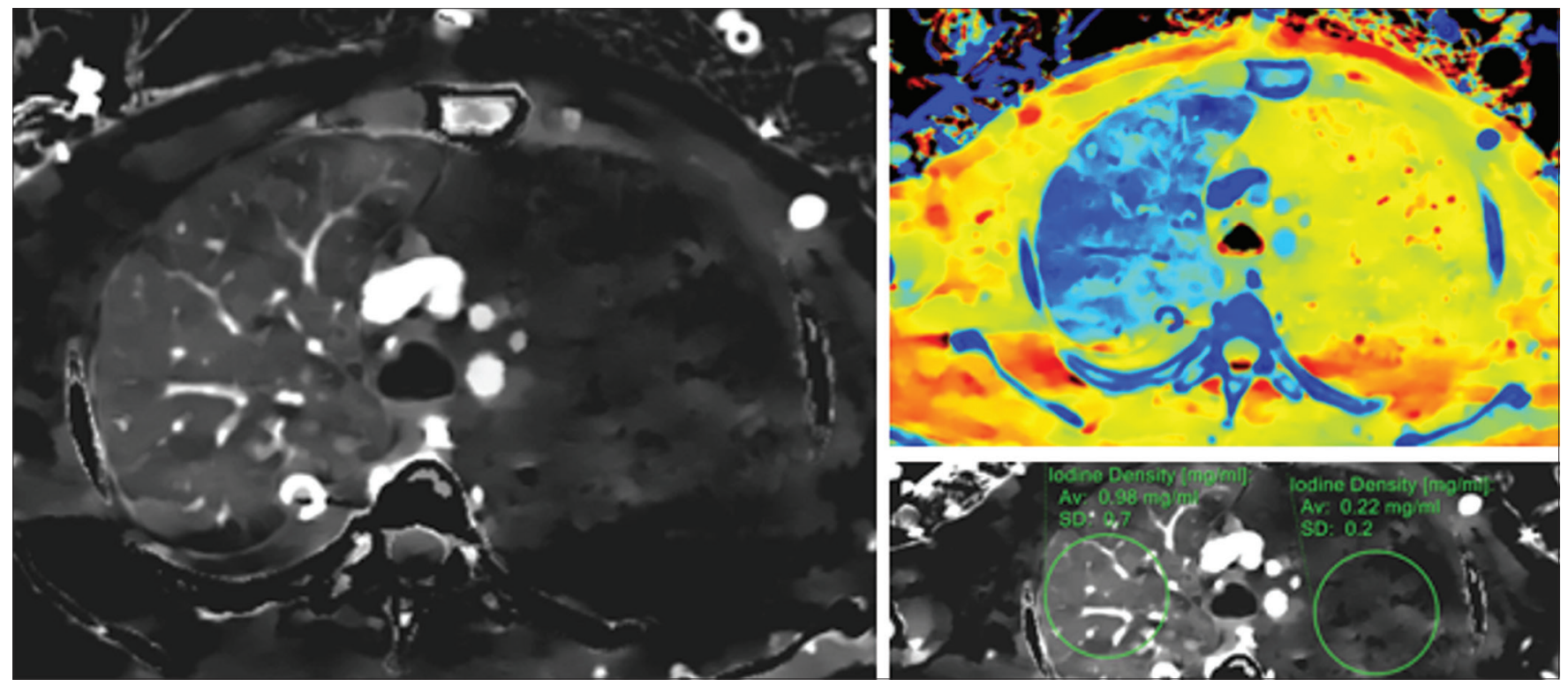

Figure 2: Dual energy reconstruction of the imaging study on day six. Axial images of iodine map (left and right lower with measurements) and effective atomic number reconstructions (right upper) elegantly demonstrate the absence of perfusion within the left lung as compared to normal appearing right lung, in keeping vascular compromise and graft rejection 


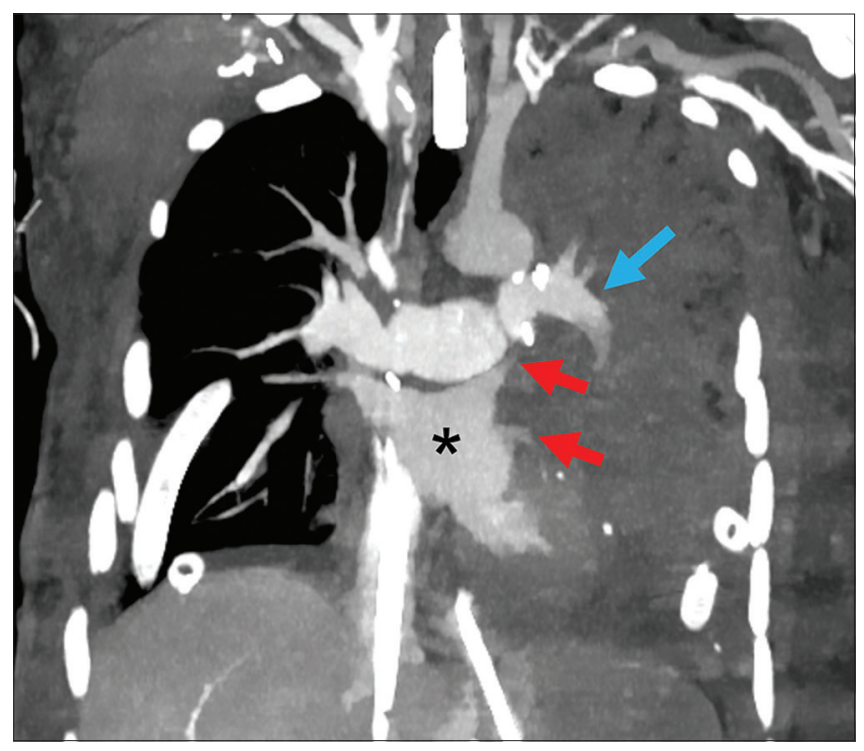

Figure 3: Paracoronal CT image of the chest demonstrating complete occlusion of left superior and inferior pulmonary veins just proximal to their drainage into the left atrium (red arrows). Proximal left side central pulmonary arteries were found to be patent (blue arrow); however, thrombosis extended to some peripheral arteries. Left atrium is within normal limits without intraluminal thrombus (asterisk)

ultimately leads to intimal thickening and fibrosis of graft vessels within months or years after transplantation..$^{[7,8]}$

Besides organ rejection, hematologic distortions are common. Because of higher risk for deep venous thrombosis during the postsurgical course, thrombosis of the transplanted arteries is fairly common. ${ }^{[9]}$ In addition to this, venous occlusion is a known risk after lung transplantation-associated risk factors comprise age, diabetes, and pneumonia. ${ }^{[1,6]}$

Differential diagnosis for extensive airspace consolidations in a transplanted lung comprise severe pulmonary edema (cardiogenic or reperfusion), acute rejection, and infection. All except infection should have maintained or slightly decreased perfusion to the lung on iodine density maps. Complete absence of perfusion highly favored acute rejection and warranted relook at the vascular structures of the lung.

In line with an earlier study demonstrating utility of iodine maps for assessment of pulmonary embolism, here, iodine maps were helpful in raising conspicuity of a perfusion defect within the left lung, and thus, other differentials became more unlikely in light of this additional information. ${ }^{[10]}$

Novel spectral detector computed tomography allows for computation of iodine maps which can be helpful in assessment of organ perfusion after transplantation; here, they illustrate pulmonary venous occlusion after lung transplantation.

\section{Declaration of patient consent}

The authors certify that they have obtained all appropriate patient consent forms. In the form the patient(s) has/have given his/her/their consent for his/her/their images and other clinical information to be reported in the journal. The patients understand that their names and initials will not be published and due efforts will be made to conceal their identity, but anonymity cannot be guaranteed.

\section{Financial support and sponsorship}

Parts of this study have been funded under a research agreement between Case Western Reserve Universtiy, University Hospitals Cleveland Medical Center and Philips Healthcare.

\section{Conflicts of interest}

Nils Große Hokamp is on the speaker's bureau of Philips Healthcare.

\section{References}

1. Yegen HA, Lederer DJ, Barr RG, Wilt JS, Fang Y, Bagiella E, et al. Risk factors for venous thromboembolism after lung transplantation. Chest 2007;132:547-53.

2. Saito H, Noda K, Ogasawara K, Atsuji S, Takaoka H, Kajihara H, et al. Usefulness and limitations of dual-layer spectral detector computed tomography for diagnosing biliary stones not detected by conventional computed tomography: A report of three cases. Clin J Gastroenterol 2018;11:172-7.

3. McCollough CH, Leng S, Yu L, Fletcher JG. Dual- and Multi-Energy CT: Principles, technical approaches, and clinical applications. Radiology 2015;276:637-53.

4. Jacobsen MC, Schellingerhout D, Wood CA, Tamm EP, Godoy MC, Sun J, et al. Intermanufacturer comparison of dual-energy CT iodine quantification and monochromatic attenuation: A phantom study. Radiology 2018;287:224-34.

5. Oda S, Nakaura T, Utsunomiya D, Hirakawa K, Takashio S, Izumiya $\mathrm{Y}$, et al. Late iodine enhancement and myocardial extracellular volume quantification in cardiac amyloidosis by using dual-energy cardiac computed tomography performed on a dual-layer spectral detector scanner. Amyloid 2018;1-2. doi: 10.1080/13506129.2018.1424625.

6. Lee JC, Christie JD. Primary craft dysfunction. Proc Am Thorac Soc 2009;6:39-46.

7. Moreau A, Varey E, Anegon I, Cuturi M-C. Effector mechanisms of rejection. Cold Spring Harb Perspect Med 2013;3:1-33.

8. Rocha PN, Plumb TJ, Crowley SD, Coffman TM. Effector mechanisms in transplant rejection. Immunol Rev 2003;196:51-64.

9. Sáez-Giménez B, Berastegui C, Loor K, López-Meseguer M, Monforte $\mathrm{V}$, Bravo $\mathrm{C}$, et al. Deep vein thrombosis and pulmonary embolism after solid organ transplantation: an unresolved problem. Transplant Rev (Orlando) 2015;29:85-92.

10. Wu HW, Cheng JJ, Li JY, Yin Y, Hua J, Xu JR. Pulmonary embolism detection and characterization through quantitative iodine-based material decomposition images with spectral computed tomography imaging. Invest Radiol 2012;47:85-91. 